\title{
ON SOME BOUNDARY PROBLEMS IN THE THEORY OF CONFORMAL MAPPINGS OF JORDAN DOMAINS
}

\author{
KIKUJI MATSUMOTO
}

1. It is a well-known result in the theory of conformal mappings of Jordan domains that if a domain $D$ in the $z$-plane bounded by a closed Jordan curve $C$ is mapped conformally on the disc $|w|<1$ by a function $w=f(z)$, analytic and univalent in $D$, then $f(z)$ will be continuous on the closure of $D$ and will map $C$ on $|w|=1$ in a one to one manner (Carathéodory [2]), and that if $C$ is rectifiable, then $f(z)$ will map sets $E$ of points of linear measure zero on $C$ onto sets of linear measure zero on the circumference $|w|=1$ and sets $E$ of positive linear measure onto sets of positive linear measure on $|w|=1$ (F. and M. Riesz [12] and Lusin and Privaloff [8]). If the condition that $C$ is rectifiable is dropped, however, the above metric property can no longer be asserted for $f(z)$ on $C$. In fact, Lavrentieff gives in his paper [5] an example of a domain $D$ bounded by a non-rectifiable closed Jordan curve $C$, by the conformal map $w=f(z)$ of which on the unit disc $|w|<1$ a set $E$ of linear measure zero on $C$ is mapped onto a set of positive linear measure on $|w|=1$ and Lohwater and Seidel [6] and Lohwater and Piranian [7] show that there exist Jordan domains $D$, by the conformal map $w=f(z)$ of which on $|w|<1$ a set $E$ of positive linear or two-dimensional measure on $C$ is mapped onto a set of linear measure zero on $|w|=1$. R. Nevanlinna [10; p. 107] also states without proof that an example of a set $E$ can be given which belongs to the boundaries of two Jordan domains $D_{1}$ and $D_{2}$ and is mapped onto a set of linear measure zero by the conformal map of $D_{1}$ on the unit disc, while it is mapped onto a set of positive linear measure by the map of $D_{2}$ on the unit disc. Here we raise the following problems:

(i) Under what metrical condition for $E$ can the condition that $C$ is rectifiable be dropped to assert that it is mapped onto a set of linear measure zero?

(ii) Under what metrical condition for $E$ can the condition that $C$ is recti-

Received November 15, 1963. 
fiable be dropped to assert that it is mapped onto a set of positive linear measure?

The main purpose of this paper is to give some answers for these problems. In the sequel, proving two elementary lemmas, we shall show that the condition that the $1 / 2$-dimensional Hausdorff measure of $E$ is zero is sufficient for the problem (i). These two lemmas can also give a partial answer for the so-called Beurling conjecture on Fuchsian or Fuchsoid groups. We shall prove that, if $E$ is of $1 / 2$-dimensional Hausdorff measure zero, then the conjecture holds good.

For the problem (ii), we shall show that, for any totally disconnected compact set $E$ of the $z$-plane, there can be found a Jordan domain $D$ such that $E$ belongs to the boundary of $D$ and is mapped onto a set of logarithmic capacity zero, consequently of linear measure zero, by the conformal map of $D$ on the unit disc. In this connection, we shall give an example of $E$ of positive $\alpha$ capacity $(0<\alpha<1)$ which belongs to a rectifiable Jordan curve $C$ and is mapped onto a set of logarithmic capacity zero by the conformal map of the Jordan domain bounded by $C$ on the unit disc. In the case where $0<\alpha<1 / 2$, such an example was given already by Ohtsuka in his paper [11] and he raised there the question whether the similar example can be given in the case where $1 / 2$ $\leqq \alpha<1$.

2. First we shall be concerned with the problem (i) raised above. Let $E$ be a totally disconnected compact set in the $z$-plane and let $C$ be a closed Jordan curve which passes every point of $E$ and bounds a domain $D$. (By Theorem 1 in Moore and Kline [9], such a Jordan curve always exists.)

We consider the class $O_{A B}^{0}$ of open Riemann surfaces, any subregion $G$ of which admits no non-constant single-valued bounded analytic function with real part vanishing continuously on its relative boundary, that is, $G \in S O_{A B}$ in notation (Kuroda [4]). Here a subregion $G$ of a Riemann surface $R$ means a subdomain of $R$ with relative boundary clustering nowhere in $R$, each point of which is regular for the Dirichlet problem with respect to $G$. Noticing that if $G$ is simply-connected, the condition that $G \in S O_{A B}$ implies that $G$ admits no nonconstant bounded harmonic function vanishing continuously on its relative boundary, we see that

if the complementary domain of $E$ with respect to the extended $z$-plane belongs to the class $O_{A B}^{0}$, it is mapped onto a set of linear measure zero on $|w|=1$ 
by the conformal map of $D$ on $|w|<1$ for any $C$, rectifiable or non-rectifiable.

By a criterion of Kuroda [4] for a Riemann surface to belong to the class $O_{A B}^{0}$, we have the following some metrical condition from the above function theoretic one.

THEOREM 1. If there exists a sequence of ring domains $A_{n, k}(n=1,2, \ldots$; $k=1,2, \ldots, \nu(n))$ such that, for each $n$, all of $A_{n+1, k}(k=1,2, \ldots, \nu(n+1))$ together separate $E$ from all of $A_{n, k}(k=1,2, \ldots, \nu(n))$ and

$$
\lim _{N \rightarrow \infty} \sup \left\{\sum_{n=1}^{N} \log \mu_{n}-\log \nu(N)\right\}=\infty,
$$

then $E$ is mapped onto a set of linear measure zero on $|w|=1$ by the conformal map of $D$ on $|w|<1$ for any $C$, rectifiable or non-rectifiable. Here $\mu_{n}$ denotes the minimum harmonic modulus of $A_{n, k}(k=1,2, \ldots, \nu(n))$.

3. In this section, we shall give two elementary lemmas in order to give a purely metrical condition concerning the problem (i).

Let $\delta$ be the unit disc $|z|<1$ and let $\rho_{j}(j=1,2, \ldots, n)$ and $\rho$ be radial segments $a_{j} \leqq r \leqq b_{j}, \theta=\theta_{j}(j=1,2, \ldots, n)$ and the union of radial segments $a_{j} \leqq r \leqq b_{j}, \theta=0(j=1,2, \ldots, n)$ respectively, where $z=r e^{i \theta}, 0<a_{1}<b_{1} \leqq a_{2}<b_{2}$ $\leqq \cdots \leqq a_{n}<b_{n} \leqq 1$ and $0 \leqq \theta_{j}<2 \pi$. We denote by $\widetilde{\omega}_{p}(z)$ and $\omega_{\mathrm{p}}(z)$ the harmonic measure of $\bigcup_{j} \rho_{j}$ with respect to the domain $\delta-\bigcup_{j} \rho_{j}$ and that of $\rho$ with respect to the domain $\delta-\rho$, respectively. Then we can prove

$$
\text { LEMMA } 1 . \quad \widetilde{\omega}_{p}(0) \geqq \omega_{p}(0),
$$

where the equality holds if and only if $\bigcup_{j} \rho_{j}$ coincides with $\rho$ or some rotation of $\rho$ around the origin.

Proof. Suppose that $b_{n}<1$. If we define the value of $\widetilde{\omega}_{p}$ at each point of $\bigcup_{j} \rho_{j}$ by 1 , then $\widetilde{\omega}_{\rho}$ is continuous and superharmonic in $\delta$. Obviously, this superharmonic $\widetilde{\omega}_{p}$ is a Green potential in $\delta$, so that it can be represented by a suitable positive mass-distribution $\mu$ on $\bigcup_{j} \rho_{j}$ as follows:

$$
\widetilde{\omega}_{p}(z)=\int_{\delta} \log \left|\frac{1-\bar{\zeta} z}{z-\zeta}\right| d \mu(\zeta)
$$

We now define the mass-distribution $\nu$ on $\delta$ by 


$$
d_{\nu}(\zeta)= \begin{cases}d_{\mu}\left(e^{i \theta_{j} \zeta}\right) & \text { if } \arg \zeta=0 \text { and } a_{j} \leqq|\zeta| \leqq b_{j} \\ 0 & \text { if } \zeta \in \delta-\rho,\end{cases}
$$

and consider the potential

$$
U^{\nu}(z)=\int_{\delta} \log \left|\frac{1-\bar{\zeta} z}{z-\zeta}\right| d \nu(\zeta) .
$$

Then this is non-negative in $\delta$ and harmonic in $\delta-\rho$. Further it is continuous in $\delta$ because

$$
U^{\nu}(z)=\sum_{j=1}^{n} \int_{\rho,} \log \left|\frac{1-\bar{\zeta} e^{i \theta_{j}} z}{e^{i \theta_{j} z}-\zeta}\right| d \mu(\zeta)
$$

on the one hand and each term of the right side is continuous in $z$ on the other. Next we compare the value of $U^{\nu}$ at a point $z, a_{j} \leqq|z| \leqq b_{j}$. of $\rho$ with the value of $\widetilde{\omega}_{p}$ at the point $e^{i \theta_{j}} z \in \rho_{j}$. Since

$$
\log \left|\frac{1-\bar{\zeta} e^{i \theta_{j}} z}{e^{i \theta_{j}} z-\zeta}\right| \leqq \log \left|\frac{1-\bar{\zeta} e^{i \theta k} z}{e^{i \theta k} z-\zeta}\right| \quad \text { for } \zeta \in \rho_{k},
$$

we have

$$
U^{\nu}(z) \geqq \sum_{k=1}^{n} \int_{\rho_{k}} \log \left|\frac{1-\bar{\zeta} e^{i \theta_{j}} z}{e^{i \theta_{j}} z-\zeta}\right| d \mu(\zeta)=\widetilde{\omega}_{\mathrm{p}}\left(e^{i \theta_{j}} z\right)=1
$$

Consequently, $U^{\prime \prime}(z)$ is not smaller than 1 at every point of $\rho$ and hence

$$
U^{\nu}(z) \geqq \omega_{\rho}(z) .
$$

Noticing that

$$
U^{\nu}(0)=\sum_{j=1}^{n} \int_{\rho_{j}} \log \left|\frac{1}{\zeta}\right| d \mu(\zeta)=\widetilde{\omega}_{\rho}(0),
$$

we have thus

$$
\widetilde{\omega}_{p}(0) \geqq \omega_{p}(0) .
$$

As the limiting case as $b_{n} \rightarrow 1$, the same holds in the case where $b_{n}=1$.

The assertion concerning the equality can be easily seen from the above argument. The proof is now complete.

Next lemma is

Lemma 2. Let $G(r, \ell)(0<r<\ell)$ denote the domain obtained by deleting the closed disc $|z| \leqq r$ and the segment on the real axis $r \leqq x \leqq \ell, y=0 \quad(z=$ $x+i y)$ from the extended $z$-plane. Then the harmonic measure $\omega(z ; r, \ell)$ of 
the circumference $|z|=r$ with respect to the domain $G(r, \ell)$ satisfies that

$$
\omega(\infty ; r, \ell)=O\left(\sqrt{\frac{r}{\ell}}\right)
$$

for every sufficiently small $r / \ell$.

Proof. We operate to the domain $G(r, \ell)$ transformations

$$
z_{1}=\frac{z}{r}, \quad z_{2}=\frac{1}{z_{1}}, \quad z_{3}=\frac{z_{2}-r / \ell}{1-(r / \ell) z_{2}}, \quad z_{4}=\sqrt{z_{3}} \text { and } \zeta=\left(\frac{1+z_{4}}{1-z_{4}}\right)^{2}
$$

one after another. Then the domain $G(r, \ell)$ is mapped conformally on the upper-half plane $\Im \zeta>0$ in the following manner:

(1) the circumference $|z|=r$ corresponds to the negative real axis of the $\zeta$-plane,

(2) the point at infinity $z=\infty$ corresponds to the point

$$
\zeta=\left(\frac{1+i \sqrt{r / \ell}}{1-i \sqrt{r / \ell}}\right)^{2}
$$

Now suppose that $r / \ell$ is sufficiently small. Then

$$
\left(\frac{1+i \sqrt{r / \ell}}{1-i \sqrt{r / \ell}}\right)^{2} \sim 1+4 i \sqrt{\frac{r}{\ell}}
$$

so that the value of the harmonic measure of the negative real axis with respect to the upper-half plane $\Im \zeta>0$ at the point $\zeta=[(1+i \sqrt{r / \ell}) /(1-i \sqrt{r / \ell})]^{2}$ is approximately equal to

$$
\frac{1}{\pi} \operatorname{Arctan} 4 \sqrt{\frac{r}{\ell}} \sim \frac{4}{\pi} \sqrt{\frac{r}{\ell}} .
$$

Hence we can conclude by (1) and (2) that

$$
\omega(\infty ; r, \ell) \sim \frac{4}{\pi} \sqrt{\frac{r}{\ell}} .
$$

Our lemma is thus proved.

4. Denoting by $m_{\alpha}(0<\alpha \leqq 2)$ the $\alpha$-dimensional Hausdorff measure, we now prove

THEOREM 2. If

$$
m_{1 / 2}(E)=0,
$$

then it is mapped onto a set of linear measure zero on $|w|=1$ by the conformal 
map of $D$ on $|w|<1$ for any $C$, rectifiable or non-rectifiable.

Proof. For a point $z_{0}$ in $D$, the linear transformation $z^{\prime}=1 /\left(z-z_{0}\right)$ maps $D$ on a domain $D^{\prime}$ containing the point at infinity. We note that under this transformation, the property of a compact set $K \notin z_{0}$ in the $z$-plane that $m_{1 / 2}(K)$ $=0$ is preserved, so that the transform $E^{\prime}$ of $E$ satisfies that

$$
m_{1 / 2}\left(E^{\prime}\right)=0 .
$$

Let $2 \ell$ denote the diameter of the transform $C^{\prime}$ of $C$ and let $r$ be a positive small number, for which the assertion of Lemma 2 holds good. Let $\varepsilon$ be a positive number, arbitrarily small. Then by the definition of the 1/2-dimensional Hausdorff measure, there exists a finite number of compact discs $\delta_{i}(i=1,2$, $\ldots, n)$ in the $z^{\prime}$-plane such that

(1) the radius $r_{i}$ of any $\delta_{i}$ is smaller than $r$,

(2) their union $\bigcup_{i=1}^{n} \delta_{i}$ covers $E^{\prime}$,

(3) $\sum_{i=1}^{n} \sqrt{r_{i}}<\varepsilon \sqrt{\ell}$.

We denote by $D^{\prime}(i)(i=1,2, \ldots, n)$ the connected component of the open set $D^{\prime}-\delta_{i}$ which contains the point at infinity and by $D^{\prime}(\infty)$ that of the open set $D^{\prime}-\bigcup_{i=1}^{n} \delta_{i}$. Further, we denote by $\omega_{i}\left(z^{\prime}\right)(i=1,2, \ldots, n)$ and $\omega_{\infty}\left(z^{\prime}\right)$ the harmonic measure of the part of the boundary of $D^{\prime}(i)$ contained in the circumference $c_{i}$ of $\delta_{i}$ with respect to $D^{\prime}(i)$ and that of the part of the boundary of $D^{\prime}(\infty)$ contained in $\bigcup_{i=1}^{n} c_{i}$ with respect to $D^{\prime}(\infty)$, respectively. Then

$$
\omega_{\infty}\left(z^{\prime}\right) \leqq \sum_{i=1}^{n} \omega_{i}\left(z^{\prime}\right) \quad \text { in } D^{\prime}(\infty) .
$$

Now we estimate each $\omega_{i}(\infty)$. Denoting by $z_{i}^{\prime}$ the centre of $c_{i}$, we consider for any $r_{i}^{\prime}, r_{i}<r_{i}^{\prime}<\ell$, the part $C_{i}^{\prime}$ of $C^{\prime}$ lying outside of the disc $\left|z^{\prime}-z_{i}^{\prime}\right| \leqq r_{i}^{\prime}$. Since the diameter of $C^{\prime}$ is $2 \ell, C_{i}^{\prime}$ contains at least one continuum $\gamma_{i}$ which joins two circles $\left|z^{\prime}-z_{i}^{\prime}\right|=r_{i}^{\prime}$ and $\left|z^{\prime}-z_{i}^{\prime}\right|=\ell$. Let $\Delta_{i}$ be the complementary domain of $\gamma_{i}$ with respect to the extended $z^{\prime}$-plane and let $\left\{\Delta_{i, k}\right\}_{k=0,1,2, \ldots}$ be a normal exhaustion of $\Delta_{i}$ such that $\Delta_{i, 0} \supset \delta_{i}$. Then the harmonic measure $w_{i, k}\left(z^{\prime}\right)$ of $c_{i}$ with respect to $\Delta_{i, k}-\delta_{i}$ converges as $k \rightarrow \infty$ uniformly on each relatively compact subset of $\Delta_{i}-\delta_{i}$ to that $w_{i}\left(z^{\prime}\right)$ with respect to $\Delta_{i}-\delta_{i}$ and

$$
\omega_{i}\left(z^{\prime}\right) \leqq w_{i}\left(z^{\prime}\right) \quad \text { in } D^{\prime}(i) .
$$

Therefore for any $\varepsilon^{\prime}>0$, arbitrarily small, there exists a $k$ such that 


$$
\omega_{i}(\infty)-\varepsilon^{\prime}<w_{i, k}(\infty) .
$$

The complement $\mathscr{C} \bar{\Delta}_{i, k}$ of $\bar{\Delta}_{i, k}$ is an open set containing $\gamma_{i}$ and hence we can find in $\mathscr{C} \bar{\Delta}_{i, k}$ a finite set of segments $\rho_{j}: a_{j} \leqq\left|z^{\prime}-z_{i}^{\prime}\right| \leqq a_{j_{+1}}$, arg $\left(z^{\prime}-z_{i}^{\prime}\right)=\theta_{j}$ $(j=1,2, \ldots, m)$, where $a_{1}=r_{i}^{\prime}<a_{2}<\cdots<a_{m}=\ell$. Map the outside of $c_{i}$ on the unit disc $|\zeta|<1$ by $\zeta=r_{i} /\left(z^{\prime}-z_{i}^{\prime}\right)$ and use Lemma 1 . Then we see that

$$
w_{i, k}(\infty) \leqq w_{p}(\infty)<\widetilde{w}_{p}(\infty),
$$

where $w_{\rho}\left(z^{\prime}\right)$ and $\widetilde{w}_{\rho}\left(z^{\prime}\right)$ are the harmonic measures of $c_{i}$ with respect to the domain $\left\{\left|z^{\prime}-z_{i}^{\prime}\right|>r_{i}\right\}-\bigcup_{j-1}^{m} \rho_{j}$ and the domain $\left\{\left|z^{\prime}-z_{i}^{\prime}\right|>r_{i}\right\}-\rho\left(\rho: r_{i}^{\prime} \leqq\left|z^{\prime}-z_{i}^{\prime}\right| \leqq \ell\right.$, $\left.\arg \left(z^{\prime}-z_{i}^{\prime}\right)=0\right)$, respectively. Because of the arbitrariness of $\varepsilon^{\prime}>0$, we have thus

$$
\omega_{i}(\infty) \leqq \widetilde{w}_{p}(\infty)
$$

and have

$$
\omega_{i}(\infty) \leqq \omega\left(\infty ; r_{i}, \ell\right)
$$

as the limiting case as $r_{i}^{\prime} \rightarrow r_{i}$, for in this case $\rho$ is the segment $r_{i} \leqq\left|z^{\prime}-z_{i}^{\prime}\right| \leqq \ell$, $\arg \left(z^{\prime}-z_{i}^{\prime}\right)=0$ and hence the domain $\left\{\left|z^{\prime}-z_{i}^{\prime}\right|>r_{i}\right\}-\rho$ is conformally equivalent to the domain $G\left(r_{i}, \ell\right)$ in the manner that the points at infinity correspond each other. By Lemma 2 we see that

$$
\omega_{i}(\infty)=O\left(\sqrt{\frac{r_{i}}{\ell}}\right)
$$

so that, by (3),

$$
\omega_{\infty}(\infty) \leqq \sum_{i=1}^{n} \omega_{i}(\infty)=O\left(\sum_{i=1}^{n} \sqrt{r_{i} / \ell}\right)=O(\varepsilon) .
$$

Hence it follows that, at the point corresponding to $z=z_{0}$, the harmonic measure of the image of $E$ on $|w|=1$ with respect to the unit disc $|w|<1$ take a value $O(\varepsilon)$ for arbitrarily small $\varepsilon$, that is, zero. Thus we can conclude that the image of $E$ on $|w|=1$ is of linear measure zero, and our theorem is established.

It is an open question whether the complementary domain of a compact set $E$ of $1 / 2$-dimensional Hausdorff measure zero belongs to the class $O_{A B}^{0}$. If this question is answered in the positive, Theorem 2 follows immediately from the fact stated above Theorem 1 .

5. Let $E$ be a compact set in the $z$-plane which contains at least three 
points and has the complementary domain $\Omega$ with respect to the extended $z$ plane. We consider the Fuchsian or Fuchsoid group $\&$ which corresponds to the Decktransformationsgruppe of the universal covering surface of $\Omega$ and denote by $N$ a normal polygon in the unit disc, which is a fundamental domain of 8 . The point set on the unit circumference of the closure of $N$ is called the foot of the normal polygon $N$. Then Beurling's conjecture says that the linear measure of the foot is zero for any $E$ of the class $W$ in Kametani's sense [3] ( = the class $N_{\mathfrak{B}}$ in Ahlfors-Beurling's sense [1]).

It is well-known that if $E$ is of logarithmic capacity zero, then the foot is of linear measure zero. Now we prove

THEOREM 3. If $m_{1 / 2}(E)=0$, then the foot is of linear measure zero.

Proof. Let $F$ denote the foot of the normal polygon $N$. Then the complement of $F$ with respect to the unit circumference $|w|=1$ consists of an at most countable number of open arcs $\left\{\alpha_{k}\right\}$. Denoting by $\beta_{k}$ the circular arc in $|w|<1$ which is orthogonal to $|w|=1$ and has the common end points with $\alpha_{k}$, we consider the domain $\hat{N}$ whose boundary consists just of $\bigcup_{k} \beta_{k}$ and $F$. Then we observe that $\tilde{N}$ is a subdomain of $N$, because $N$ is convex relative to the hyperbolic metric, and that the point set on $|w|=1$ of the closure of $\tilde{N}$ coincides with $F$. Now we denote by $D$ the domain in the extended $z$-plane which corresponds to the normal polygon $\hat{N}$. Then the boundary of $D$ consists of $E$ and an at most countable number of analytic $\operatorname{arcs}\left\{\gamma_{i}\right\}$ clustering nowhere in the complementary domain $\Omega$ of $E$. Now we map $D$ on the unit disc $|\zeta|<1$ by a conformal map $\zeta=\zeta(z)$, which is continuable analytically beyond each side of $\gamma_{i}$, and see that every point of $U_{\gamma_{i}}$ corresponds to just two points of the unit circumference $|\zeta|=1$. From the same argument as in the proof of Theorem 2, we see that the set $E_{\zeta}$ of points on $|\zeta|=1$, which has no corresponding point on $U_{\gamma_{i}}$, is of linear measure zero.

Denote by $z=\varphi(w)$ the conformal map of $\tilde{N}$ on the domain $D$. Then the map $\zeta=\zeta(\varphi(w))$ maps conformally the Jordan domain $\tilde{N}$ on the unit disc $|\zeta|<1$ in the manner that $F$ is mapped onto $E_{\zeta}$. Since the Jordan curve bounding $\tilde{N}$ is rectifiable and the linear measure of $E_{\zeta}$ is zero, we can now conclude by F. and M. Riesz' theorem that $F$, that is, the foot of $N$ is of linear measure zero.

6. As an answer for the problem (ii), we shall prove the following theorem. 
This shows that some conditions on $C$ other than the metrical conditions on $E$ are needed in order that $E$ is mapped onto a set of positive linear measure.

THEOREM 4. For any totally disconnected compact set $E$ in the z-plane, there exists a Jordan domain $D$ such that the Jordan curve $C$ bounding $D$ passes every point of $E$ and $E$ is mapped onto a set of logarithmic capacity zero by the conformal map of $D$ on the unit disc.

Proof. By Theorem 1 in Moore and Kline [9], there exists a Jordan curve $\Gamma$ which passes throgh every point of $E$. We can map conformally the domain bounded by $\Gamma$ on the upper-half $\zeta$-plane, $3 \zeta>0$, in such a way that the image $E_{\zeta}$ of $E$ on the real axis of the $\zeta$-plane is compact. Then $E_{\zeta}$ is a totally disconnected compact set. Now we shall prove that there is a Jordan curve $C_{\zeta}$ in $\Im \zeta \geqq 0$, which passes through every point of $E_{\zeta}$, and the domain bounded by it is mapped on the unit disc $|w|<1$ in the manner that the image of $E_{\xi}$ on $|w|=1$ is of capacity zero. If this is proved, it is enough for us only to take as $D$ the domain in the $z$-plane bounded by the Jordan curve $C$ which corresponds to $\mathrm{C}_{\zeta}$.

Without any loss of generality, we may suppose that $E_{\zeta}$ is contained in $-1 / 2 \leqq \xi \leqq 1 / 2(\zeta=\xi+i \eta)$. First we cover $E$ by a finite number of open squares $\left\{\delta_{1 i}\right\}$ with center on $\eta=0$ and with sides of length less than $1 / 4$. Calling each region of the type

$$
a<\xi<a+d, b \leqq \eta \leqq c
$$

a vertical strip with width $d$, we join the domain $D_{v},-1<\xi<1,1 / 2<\eta<1$ and each connected component of $\bigcup \delta_{1 i}$ with a vertical strip $s_{1 j}$ with width so narrow that

$$
\sum_{j} D\left(\omega_{1 j}\right) \leqq 1
$$

where $\omega_{1 j}$ is the harmonic function in $s_{1 j}$ such that

$$
\omega_{1 j}=\left\{\begin{array}{lll}
0 & \text { on } & s_{1 j} \cap \bar{D}_{0} \\
1 & \text { on } & s_{1 j} \cap \overline{\left(\cup \delta_{1 i}\right)}
\end{array}\right.
$$

and its normal derivative on the vertical sides of $s_{1 j}$ is zero. We denote by $2 \varepsilon_{1}$ the minimum length of sides of squares $\delta_{1 i}$ and by $D_{1}$ the simply-connected domain 


$$
\left[D_{0} \cup\left(\bigcup_{j} s_{1 i}\right) \cup\left(\bigcup_{i} \delta_{1 i}\right)\right] \cap\left\{\eta>\varepsilon_{1} / 2\right\} .
$$

Next we cover $E_{\zeta}$ by a finite number of open squares $\left\{\delta_{2 i}\right\}$ with center on $\eta=0$ and with sides of length less than $\varepsilon_{1} / 4$, each of which is contained in some $\delta_{1 i}$, and join $D_{1}$ and each connected component of $U \delta_{2 i}$ with a vertical strip $s_{2 j}$ with width so narrow that

$$
\sum_{j} D\left(\omega_{2 j}\right) \leqq 1
$$

where $\omega_{2 j}$ is the same one for $s_{2 j}$ as $\omega_{1 j}$ for $s_{1 j}$. Denoting by $2 \varepsilon_{2}$ the minimum length of sides of squares $\delta_{2 i}$, we define the domain $D_{2}$ by

$$
D_{2}=\left[D_{1} \cup\left(\bigcup_{j} s_{2 j}\right) \cup\left(\bigcup_{i} \delta_{2 i}\right)\right] \cap\left\{\eta>\varepsilon_{2} / 2\right\} .
$$

Then $D_{2}$ is simply-connected, because each $s_{2 j}$ is also contained in some $\delta_{1 i}$. Defining inductively, we thus obtain an increasing sequence of domains $\left\{D_{n}\right\}$. We now set

$$
D_{\zeta}=\lim _{n \rightarrow \infty} D_{n}
$$

Then it is easily seen that $D_{\zeta}$ is a Jordan domain with a boundary curve $C_{\zeta} \supset E_{\zeta}$. So it remains for us to prove that $D_{\zeta}$ is mapped conformally on the unit disc in such a way that the image of $E_{\zeta}$ on the unit circumference is of logarithmic capacity zero. But this can be proved easily. In fact, we consider the double $\hat{D}_{\zeta}$ of $D_{\zeta}$ with respect to $C_{\zeta}-E_{\zeta}$. Then $\hat{D}_{\zeta}$ is a Riemann surface of planar character. The double $\hat{s}_{n j}$ of each $s_{n j}$ is a doubly-connected closed domain in $\hat{D}_{\zeta}$ and for a fixed $n$, all of $\hat{s}_{n j}(j=1,2, \ldots, j(n))$ together separates the ideal boundary of $\hat{D}_{\zeta}$ from the domain $\hat{D}_{0}$, the double of $D_{0}$. If we denote by $\hat{\omega}_{n}$ the harmonic function on the set $\bigcup_{j} \hat{s}_{n j}$, which takes the value 0 on the boundary separating $\bigcup_{j} \hat{s}_{n j}$ from $\hat{D}_{0}$ and the value 1 on that separating, $\bigcup_{j} \hat{s}_{n j}$ from the ideal boundary of $\hat{D}_{\zeta}$, we have

$$
\frac{2 \pi}{D\left(\omega_{n}\right)} \geqq \pi
$$

by our condition $\sum D\left(\omega_{n j}\right) \leqq 1$. Hence

$$
\sum_{n=1}^{\infty} \frac{2 \pi}{D\left(\hat{\omega}_{n}\right)}=+\infty
$$

so that we see from Sario's criterion $[13]$ that $\hat{D}_{\zeta} \in O_{G}$. This shows that the 
complementary domain of the image $E_{w}$ of $E_{\zeta}$, when we map $D_{\zeta}$ conformally on the unit disc $|w|<1$, admits no Green function, that is, the logarithmic capacity of $E_{w}$ is zero. Our proof is now complete.

7. In this last section, we shall give an example of $E$ of positive $\alpha$-capacity $(0<\alpha<1)$, such that there is a rectifiable Jordan curve $C \supset E$ and it is mapped onto a set of logarithmic capacity zero by the conformal map of the Jordan domain bounded by $C$ on the unit disc. In the case where $0<\alpha<1 / 2$, such an example was given already by Ohtsuka [11].

Let $E$ be a Cantor set on the closed interval $I_{0}:[-1 / 2,1 / 2]$ with constant successive ratios $\xi_{n}, 0<\xi_{n}=2 \ell<1$. Then for any $\alpha(0<\alpha<1), E$ is of positive $\alpha$-capacity, if we take $\ell$ sufficiently near $1 / 2$. We shall show that there is a rectifiable Jordan curve $C \supset E$ satisfying the condition.

Defining the Cantor set $E$, we repeat successively to exclude an open segment from the middle of another segment and there remain $2^{n}$ segments of equal length $\ell^{n}$ after, beginning with the interval $I_{0}$, we repeat $n$ times. We donote these segments by $I_{n, k}\left(n=1,2, \ldots ; k=1,2, \ldots, 2^{n}\right)$ and the middle point of $I_{n, k}$ by $x_{n ; k}$. Further we denote by $\delta_{n, k}$ the square with center at $x_{n, k}$ and with sides of length $\ell^{n-1} / 2$. Each $\delta_{n, k}(n \geqq 2)$ is contained in some $\delta_{n-1, k}$ and, for fixed $n$, all of $\delta_{n, k}\left(k=1,2, \ldots, 2^{n}\right)$ are mutually disjoint and together cover $\bigcup_{k} I_{n, k}$, consequently $E$. First we join the domain $D_{0},-1<x<1,1 / 2<y<1$ $(z=x+i y)$ and each of $\delta_{2, k}(k=1,2,3,4)$ with a vertical strip $s_{1, k}$, which is symmetric with respect to the line $x=x_{2, k}$, with width so narrow that

$$
\sum_{k} D\left(\omega_{1, k}\right) \leqq 1,
$$

where $\omega_{1, k}$ is the same one in $\S 6$. We define the domain $D_{1}$ by

$$
D_{1}=\left[D_{0} \cup\left(\bigcup_{k} s_{1, k}\right) \cup\left(\bigcup_{k} \delta_{2, k}\right)\right] \cap\left\{y>_{\ell} / 8\right\} \text {. }
$$

Next we join the domain $D_{1}$ and each of $\delta_{4, k}\left(k=1,2, \ldots, 2^{4}\right)$ with a vertical strip $s_{2, k}$, which is symmetric with respect to the line $x=x_{4, k}$, with width so narrow that

$$
\sum_{k} D\left(\omega_{2, k}\right) \leqq 1
$$

and define the domain $D_{2}$ by

$$
D_{2}=\left[D_{1} \cup\left(\bigcup_{k} s_{2, k}\right) \cup\left(\bigcup_{k} \delta_{4, k}\right)\right] \cap\left\{y>\ell^{3} / 8\right\} .
$$


The length of the boundary part of $D_{2}$ which is not that of $D_{1}$ is less than $2^{4}\left(\ell / 4+3 \ell^{3} / 2\right)=4 \ell\left(1+6 \ell^{2}\right)$. We define domains $\left\{D_{n}\right\}$ inductively, that is, supposing that $D_{n}$ has been defined already, we join $D_{n}$ and each of $\delta_{2(n+1), k}$ $\left(k=1,2, \ldots, 2^{2(n+1)}\right)$ with a vertical strip $s_{n+1, k}$, which is symmertric with respect to the line $x=x_{2(n+1), k}$, with width so narrow that

$$
\sum_{k} D\left(\omega_{n+1, k}\right) \leqq 1
$$

and define the domain $D_{n+1}$ by

$$
D_{n+1}=\left[D_{n} \cup\left(\bigcup_{k} s_{n+1, k}\right) \cup\left(\bigcup_{k} \delta_{2(n+1), k}\right)\right] \cap\left\{y>\ell^{2 n+1} / 8\right\} .
$$

The length of the boundary part of $D_{n+1}$ which is not that of $D_{n}$ is less than $2^{2(n+1)}\left(\ell^{2 n-1} / 4+3 \ell^{2 n+1} / 2\right)=(2 \ell)^{2(n-1)} \cdot 4 \ell\left(1+6 \ell^{2}\right)$. We now set

$$
D=\lim _{n \rightarrow \infty} D_{n}
$$

Then $D$ is a Jordan domain with a boundary curve $C \supset E$. Since $E$ is of linear measure zero and the length of $C-E$ is less than

$$
L+4 \ell\left(1+6 \ell^{2}\right)\left\{1+(2 \ell)^{2}+(2 \ell)^{3}+\cdots\right\} \quad(2 \ell<1),
$$

where $L$ denotes the length of the boundary curve of $D_{1}$, we see that $C$ is a rectifiable Jordan curve. Noticing that the condition

$$
\sum_{k} D\left(\omega_{n, k}\right) \leqq 1 \quad \text { for every } n \geqq 1 \text {, }
$$

we see also by the same argument in $\S 6$ that the image of $E$ on $\mid w^{\mid}=1$ by the conformal map of $D$ on the unit disc $|w|<1$ is of logarithmic capacity zero, and thus that $D$ is one of the wanted.

\section{REFERENCES}

[1] L. V. Ahlfors and A. Beurling: Conformal invariants and function-theoretic null-sets, Acta Math., 83 (1950), 101-129.

[2] C. Carathéodory: Über die gegenseitige Beziehung der Ränder bei der konformen Abbildung des Inneren einer Jordanschen Kuve auf einen Kreis, Math. Ann., 73 (1913), 305-320.

[3] S. Kametani: On Hausdorff's measures and generalized capacities with some of their applications to the theory of functions, Jap. Journ. Math., 19 (1944-48), 217-257.

[4] T. Kuroda: On analytic functions on some Riemann surfaces, Nagoya Math. Journ., 10 (1956), 27-50. 
[5] M. Lavrentieff: Sur quelques problèmes concernant_-les fonctions univalentes sur la frontière, Rec. Math., 43 (1936), 816-846 (en russe).

[6] A. J. Lohwater and W. Seidel: An example in conformal mapping, Duke Math. Journ., 15 (1948), 137-143.

[7] A. J. Lohwater and G. Piranian: Conformal mapping of a Jordan region whose boundary has positive two-dimensional measure, Michigan Math. Journ., 1 (1952), 1-4.

[8] N. Lusin and I. Privaloff: Sur l'unicité et la multiplicité des fonctions analytiques, Ann. Sci. École Norm. Sup., 42 (1925), 143-191.

[9] R. L. Moore and J. R. Kline: On the most general plane closed set through which it is possible to pass a simple continuous arc, Ann. of Math., 20 (1918-1919), 218-223.

[10] R. Nevanlinna: Eindeutige Analytische Funktionen, Berlin, 1936.

[11] M. Ohtsuka: Théorèmes étoiles de Gross et leurs applications, Ann. Inst. Fourier, 5 (1954), 1-28.

[12] F. and M. Riesz: Über die Randwerte einer analytischen Funktion, Quatrème Congré des math. scand. à Stockholm (1916), 24-47.

[13] L. Sario: Questions d'existence au voisinage de la frontière d'une surface de Riemann, C. R. Acad. Sci. Paris 230 (1950), 269-271.

\section{Mathematical Institute}

Nagoya University 